\title{
Reengineering TARMED
}

\section{Sehr geehrter Herr Kollege Häuptli}

In Ihren Ausführungen schreiben Sie zum Thema Psychiatrie: "Damit wurde ein wesentlicher Teil der mangelhaften Abbildung der Arbeitsweise der Psychiater behoben und ihre einstige Forderung von Fr. 200.- pro Stunde aus AL und TL erfüllt.» [1]

Meines Wissens ist diese Information falsch.

Ich erinnere an den Text der Forderung im Manifest der Gesellschaften für Psychiatrie sowie für Kinder- und Jugendpsychiatrie vom Anfang dieses Jahres: «Weniger als 200 Franken die Stunde - Nie!» [2]. Die beiden Fachgesellschaften erwarten bis zur Integration ihres Tarifmodells in den TARMED «Notmassnahmen, die den Psychiatern den minimalen Stundenansatz (= Umsatz, nicht Einkommen) von Fr. 200.- ermöglichen». Dies gilt bis heute.

Die Forderung ist erst dann erfüllt, wenn der Stundenansatz im SUVA/MV/IV-Bereich sowie in jedem Kanton auf Krankenkassenebene mindestens Fr. 200.- beträgt. Dazu muss auf allen genannten Verhandlungsebenen ein Taxpunktwert von etwa $93 \mathrm{Rp}$. akzeptiert sein oder zumindest gesichert erscheinen. Aber dies ist doch nicht der Fall! Sollte ich mich - erfreulicherweise - täuschen, d.h. die Forderung wirklich erfüllt sein, wäre ich Ihnen dankbar für eine ent- sprechende Erläuterung. Sollte ich mich nicht täuschen, möchte ich Sie fragen, warum die Schweizer Ärzteschaft von der FMH in einem für uns Psychiaterinnen und Psychiater existenziellen Anliegen zum zweiten Mal falsch informiert wird. Denn bereits in der Antwort des Zentralvorstandes auf das Manifest wurde die Forderung als erfüllt erklärt [3].

Wir anerkennen, dass die bisherigen Resultate des Reengineerings einen ersten Schritt darstellen, um die Zielsetzungen von TARMED auch im Bereich Psychiatrie zu verwirklichen. Was nun endlich folgen muss, ist eine Anpassung der Produktivität an die psychiatrische Praxisrealität.

Dr. med. Rudolf Bleuler, Präsident der psychiatrisch und psychotherapeutisch tätigen Ärztinnen und Ärzte beider Appenzell sowie der Gesellschaft für Intensive Dynamische Kurzpsychotherapie der Schweiz

\section{Literatur}

1 Häuptli W. Reengineering (RE) TARMED. Schweiz Ärztezeitung 2001;82(49):2553-4.

2 Schweizerische Gesellschaft für Psychiatrie und Psychotherapie, Schweizerische Gesellschaft für Kinder- und Jugendpsychiatrie und -psychotherapie. Manifest der SGP und der SGKJPP betreffend TarMed. Schweiz Ärztezeitung 2001;82(8):383.

3 Brunner HH, Deschenaux F-X. Antwort des Zentralvorstands. Schweiz Ärztezeitung 2001;82(11):533-4.

\section{TARMED und Burnout}

Walter Häuptli, Vorsitzender VD Reengineering TARMED

Vor allem Psychiater erkranken in hoher Rate an Burnout. So belegen es verschiedene Studien. Grund dafür ist die ausgeprägte emotionale Belastung während der beruflichen Tätigkeit. Inwiefern die bisherige, innerhalb der Ärzteschaft zweifelsfrei niedrige Entlöhnung an diesem misslichen Umstand Schuld trägt, ist ungewiss. Schon eher ist anzunehmen, dass kürzere Arbeitszeit mit mehr Ausgleich vorbeugend wirken dürfte. So stellt sich die Frage, ob die Aufwertung der Psychiater durch TARMED mit Erfüllung der einstigen Forderung von Fr. 200.- pro Stunde
(Berechnungsannahme: TPW Fr. 1.-) genügt, um Burnout auf das wohl nie zu unterschreitende Ausmass abzusenken. Die betroffene Fachgruppe bezweifelt dies vehement. Im Gegenteil, sie befürchtet aufgrund des durch TARMED ungebremsten Burnouts schon mittelfristig einen rasch zunehmenden Versorgungsengpass. TARMED soll also nebst Patienten auch Ärzte gefährden.

Den Vorwurf der Gefährdung von Ärzten nehmen die Vertragsparteien ernst. Zwar gelang es im RE I aus Zeitgründen tatsächlich nicht, das Problem mit der nötigen Sorgfalt zu lösen, auch wegen der Vermutung, dass nicht nur Psychiater, sondern noch andere Fachärzte von diesem Phänomen und anderer «Berufskrankheit" betroffen sind. Die TASKFORCE RE II wird sich deshalb eingehend damit befassen und in diesem Zusammenhang auch die Jahresarbeitszeit überprüfen. 